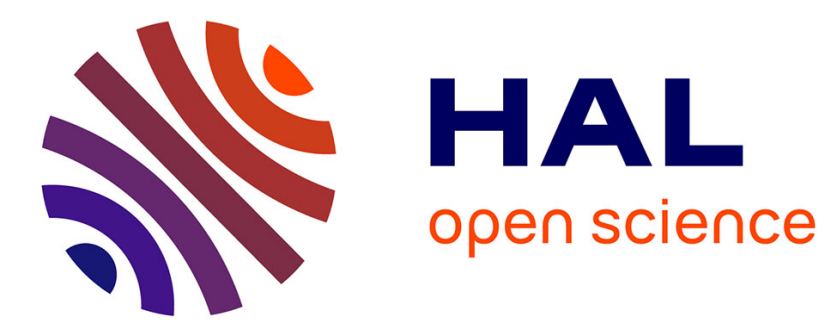

\title{
Duct modes damping through an adjustable electroacoustic liner under grazing incidence
}

Romain Boulandet, Hervé Lissek, Sami Karkar, Manuel Collet, Gaël Matten, Morvan Ouisse, Marc Versaevel

\section{- To cite this version:}

Romain Boulandet, Hervé Lissek, Sami Karkar, Manuel Collet, Gaël Matten, et al.. Duct modes damping through an adjustable electroacoustic liner under grazing incidence. Journal of Sound and Vibration, 2018, 426, pp.19-33. 10.1016/j.jsv.2018.04.009 . hal-02371016

\section{HAL Id: hal-02371016 https://hal.science/hal-02371016}

Submitted on 19 Nov 2019

HAL is a multi-disciplinary open access archive for the deposit and dissemination of scientific research documents, whether they are published or not. The documents may come from teaching and research institutions in France or abroad, or from public or private research centers.
L'archive ouverte pluridisciplinaire HAL, est destinée au dépôt et à la diffusion de documents scientifiques de niveau recherche, publiés ou non, émanant des établissements d'enseignement et de recherche français ou étrangers, des laboratoires publics ou privés.

\section{(c)(1)}

Distributed under a Creative Commons Attribution| 4.0 International License 


\title{
Duct modes damping through an adjustable electroacoustic liner under grazing incidence
}

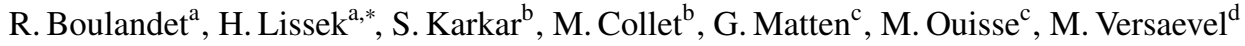 \\ ${ }^{a}$ Laboratoire de Traitement des Signaux LTS2, Ecole Polytechnique Fédérale de Lausanne, Station 11, CH-1015, Lausanne, Switzerland \\ ${ }^{b}$ LTDS, Ecole Centrale de Lyon, 36 avenue Guy de Collongue, 69131 Lyon, France \\ ${ }^{c}$ Univ. Bourgogne Franch-Comté, FEMTO-ST Institute, CNRS/UFC/ENSMM/UTBM, Department of Applied Mechanics, 24 rue de l'épitaphe, 25000 \\ Besançon, France \\ ${ }^{d}$ SAFRAN Nacelles, Route du pont 8, 76700 Gonfreville l'Orcher, France
}

\begin{abstract}
This paper deals with active sound attenuation in lined ducts with flow and its application to duct modes damping in aircraft engine nacelles. It presents an active lining concept based on an arrangement of electroacoustic absorbers flush mounted in the duct wall. Such feedback-controlled loudspeaker membranes are used to achieve locally reacting impedances with adjustable resistance and reactance. A broadband impedance model is formulated from the loudspeaker parameters and a design procedure is proposed to achieve specified acoustic resistances and reactances. The performance is studied for multimodal excitation by simulation using the finite element method and the results are compared to measurements made in a flow duct facility. This electroacoustic liner has an attenuation potential comparable to that of a conventional passive liner, but also offers greater flexibility to achieve the target acoustic impedance in the low frequencies. In addition, it is adaptive in real time to track variable engine speeds. It is shown with the liner prototype that the duct modes can be attenuated over a bandwidth of two octaves around the resonance frequency of the loudspeakers.
\end{abstract}

Keywords: Lined duct, Electroacoustic absorber, Active sound absorption, duct modes damping

\section{Introduction}

Challenging noise reduction targets, such as those specified in the European FlightPath 2050 goals, require aircraft manufacturers to develop innovative lining technologies to limit noise emission in the vicinity of airports. Since the advent of jet aircraft in commercial and private air transport, continuous efforts have been made to develop acoustic treatments and techniques to reduce engine noise. For reasons of fuel economy and noise reduction, most of today's jet airliners are powered by high-bypass turbofan engines. While this advance has resulted in much less jet exhaust noise, fan noise has become more prominent. The use of liners on the internal walls of the engine nacelles, both in the intake and by-pass ducts, play an important role in reducing fan noise before it escapes the engine, converting acoustic energy into heat. The lining specifications are usually given in terms of desired acoustic resistance and reactance, which depend on several factors related to the excitation frequency, the duct geometry and dimension, the characteristics of propagating modes, and the presence or absence of airflow through the duct [1, 2, 3]. Conventional acoustic liners such as Single Degree of Freedom (SDOF) liners consist of a thin layer of perforated plate or wire-mesh face sheet backed with a honeycomb cavity, with internal partitions closed by an impervious plate, which provide an essentially locally reacting surface. Acoustic liners properties depend essentially on the following geometric parameters: effective open area, sheet thickness, sheet thickness-to-hole diameter ratio, and honeycomb cavity depth [2, 4]. A correctly designed SDOF liner will provide an optimal attenuation around a specific grazing flow velocity at the design frequency. In the

${ }^{*}$ Corresponding author

Email address: herve.lissek@epfl.ch (H. Lissek) 
case of high-bypass turbofan engines, however, the transverse dimensions of the duct permit wave propagation of many higher-order modes.

The problem of optimizing modal sound attenuation in a lined duct has been studied by Cremer for the lowest order mode pair in a two-dimensional duct in the absence of flow [5]. Cremer's result was then extended and generalized by Tester for any mode in the case of uniform circular and annular cross-sections [6] and in the presence of uniform flow [7]. Liner resistance and reactance parameters are usually obtained experimentally, although some semi-experimental methods, referred to as impedance eduction, are available for their estimates [8, 9]. The technological challenge is therefore to design a lining capable of achieving a predetermined acoustic impedance which is effective over a wide range of engine operating conditions. The main limitations of any passive acoustic liners is that the impedance is fixed by geometry and can hardly be optimized for both the landing and take-off phases. The sound attenuation of such reactive linings is that of a sharply tuned resonator effective over a narrow frequency range. It provides excellent sound absorption over a limited bandwidth and therefore is only effective for a specific regime or flight phase. This design is a compromise between the relevant engine power ratings. Double Degree of Freedom (DDOF) liners made of two layers of honeycomb cells divided by a porous septum can be designed with a wider effective frequency range but they require higher thickness and are heavier than a single layer over their effective frequency range. In that context, it is of interest to consider how to achieve an adaptive locally reacting impedance which would allow adjusting the liner sound attenuation capability for each phase of the flight.

Different concepts relating to the application of active acoustic liners in flow ducts have been reported over the past few decades. The pioneering work in the field of active sound absorption was undertaken by Olson and May in the 1950 s by combining a loudspeaker and a microphone nearby in a negative feedback loop, known as "electronic sound absorber". As mentioned in [10], sound absorption was achieved more easily by providing a low impedance behind a dissipative acoustic impedance composed of a resistive screen. A few decades later, a similar approach was adopted by Guicking and Lorentz (1984) [11], and later by Furstoss et al. (1997) [12], to develop a hybrid passive/active treatment composed of a thin porous layer backed by an air cavity closed by a feedback-controlled loudspeaker. By successfully imposing a pressure release condition at the rear of the porous layer, a purely real surface impedance given by the flow resistance of the porous layer can be achieved and the liner behaves like an active quarter-wave resonator. Direct application of the hybrid passive/active absorber to achieve broadband noise reduction in flow ducts can be found in [13, 14, 15]. If the desired acoustic resistance for the lined duct is directly related to the porosity and thickness of the resistive layer, the reactance is generally close to zero. As discussed above, however, a purely real surface impedance condition does not lead to optimal attenuation rates of duct modes [5, 6, 7]. Alternative solutions have been proposed to implement complex acoustic impedances with the help of actively controlled electromechanical actuators. An active SDOF liner for attenuating noise that includes a rigid backplate supporting a piezoelectric patch was proposed and patented by Kraft and Kontos [16], where a microphone is used in combination with a controller to obtain a predetermined acoustic impedance at the panel surface. Zhao and Sun proposed achieving specific impedance condition actively through two controllable variables, the cavity depth of the liner and the bias flow through the orifice [17]. However, the authors concluded that change in reactance was harder to achieve practically using this approach, especially in real world conditions as it depends on the geometry of the liner. Horowitz et al. (2002) examined an actively-tuned electromechanical acoustic liner based on a Helmholtz resonator with a compliant piezoelectric composite backplate coupled to a tunable electrical shunts network. By increasing the number of degrees of freedom the filter network allows the liner to exhibit the absorption characteristics of a multi-layer lining [18]. An alternative hybrid liner based on an active Helmholtz resonator concept was suggested by Parente et al., where the objective of control is not to absorb or cancel noise but rather to scatter or redistribute acoustic energy among modes to maximize the efficiency of the passive sound absorbing elements [20]. Other promising results can be found in the literature concerning active Helmholtz resonator concepts using a loudspeaker in the resonator cavity to extend the sound absorption capability of the resonator [19]. Collet et al. (2009) investigated the potential of a distributed control scheme to block wave propagation in a given direction in a waveguide. In contrast to the active methods mentioned above, this approach aims at redirecting the sound field without directly interacting with acoustic energy to cancel or absorb it. Recently, the concept of electroacoustic absorber has been introduced as an effective means of damping the duct modes, either using shunt loudspeaker technique [22, 23], direct feedback control [24, 25], or by self-sensing control of the loudspeaker impedance [26]. Rivet et al. (2017) showed that a loudspeaker and a microphone nearby, both being connected by a model-based transfer function, can be used for matching the impedance of a loudspeaker diaphragm to a target specific acoustic impedance, which has the effect of damping the standing waves in an enclosure [27]. 
The paper presents an active lining concept based on an arrangement of electroacoustic absorbers and its application to achieve broadband noise reduction in aircraft engine nacelles. Instead of trying to improve a passive DDOF liner, emphasis is placed on achieving adjustable local reaction through active impedance control of the loudspeaker diaphragm. The theoretical analysis is based on a rectangular duct with section lined on one side in the presence of a uniform flow and a multimodal excitation. The remainder of the paper is organized as follow. The design of the baseline electroacoustic absorber from a feedback-controlled loudspeaker is addressed in Section 2 using an impedance-based approach. Section 3 provides an overview of the theoretical framework used in this study to investigate the liner performance in a flow duct. Computer simulations and experimental results are given in Section 4 to show the performance and potential of the proposed active liner. A discussion of the benefits and limitations of this active lining concept to reduce fan noise in aircraft engine nacelles is finally provided to conclude this paper.

\section{Design of the electroacoustic liner}

This section describes the design of an electroacoustic liner concept composed of distributed electroacoustic absorbers. In particular, a locally reacting impedance model is developed on the basis of the electrodynamic loudspeaker in order to achieve a given target specific acoustic impedance.

\subsection{Locally reacting impedance models}

The macroscopic properties of acoustic liners are generally characterized by the specific acoustic impedance $Z=p / v_{n}$, which defines the ratio of the local sound pressure $p$ to the normal component of the particle velocity $v_{n}$ on the lining surface. For convenience, the non-dimensional specific acoustic impedance $z$ in a lined duct is commonly given in terms of resistance $\theta$ and reactance $\chi$ as [1, 2]

$$
z=\frac{Z}{\rho c}=\theta+\mathrm{j} \chi
$$

where $\rho c$ is the characteristic impedance of air. Analytical models for predicting impedance of conventional SDOF liners are usually formulated from

$$
Z_{\text {sdof }}=R+\mathrm{j}(k m-\cot (k D))
$$

where $D$ is the lining depth, $R$ is the flow resistance of the face-sheet, $k m$ is the facing sheet inertance, $\cot (k D)$ is the cavity reactance, and $k$ is the wavenumber. In Eq. (2), acoustic resistance is mainly related to the open area of the perforated sheet while reactance strongly depends on the depth of the liner cavity. Figure 1 illustrates the schematic representation of a lined duct in the presence of a uniform mean flow.

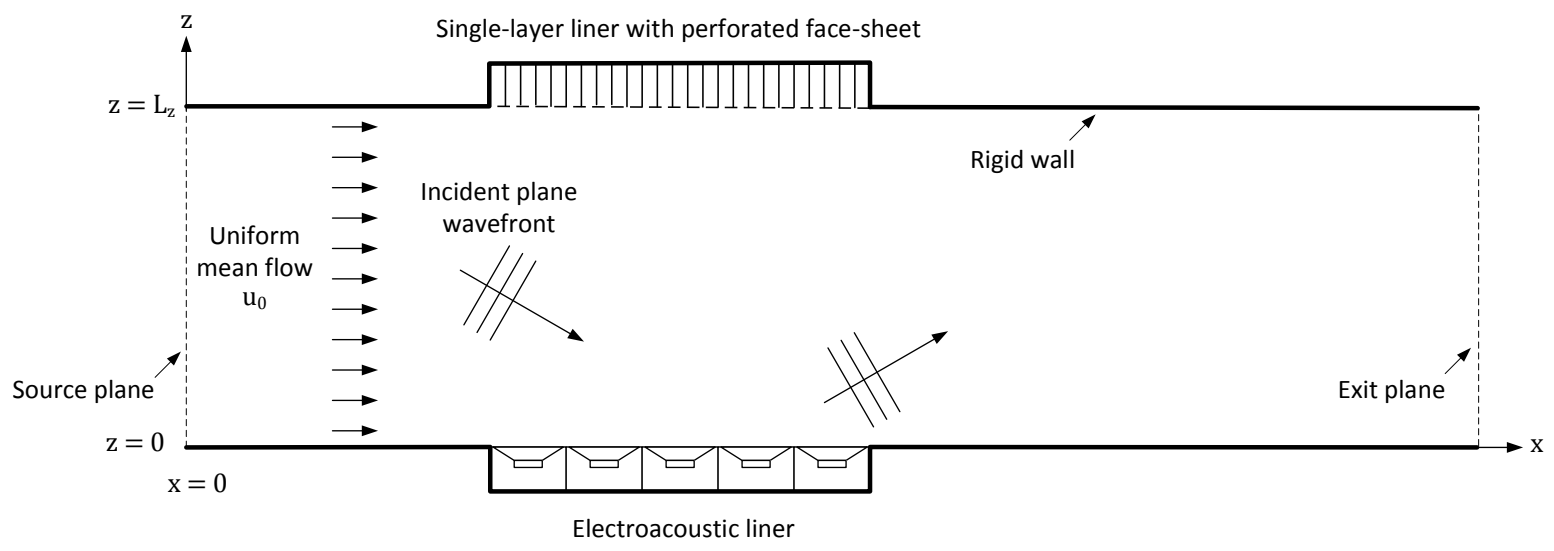

Figure 1: Schematic representation of a lined duct. 


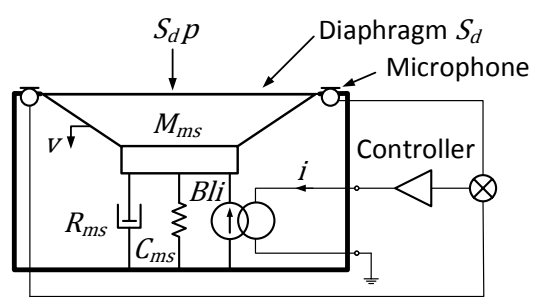

(a)

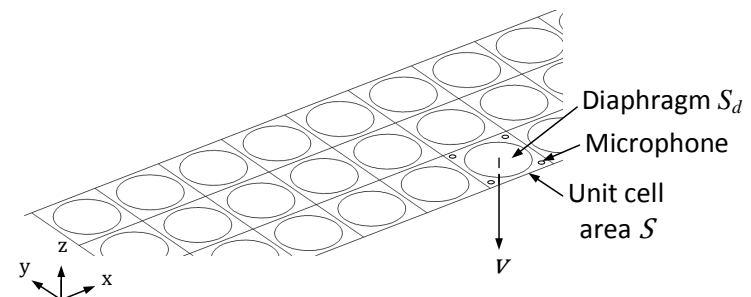

(b)

Figure 2: Schematic representation of an electrodynamic absorber (a) and electroacoustic liner concept (b).

Additionally, for a DDOF liner, the surface impedance can be expressed as [29]

$$
\frac{Z_{\mathrm{ddof}}}{\rho c}=\frac{Z_{1}}{\rho c}+\frac{\frac{Z_{2}}{\rho c} \cos \left(k d_{1}\right) \sin \left(k d_{2}\right)-\mathrm{j} \cos (k d)}{\sin (k d)+\mathrm{j} \frac{Z_{2}}{\rho c} \sin \left(k d_{1}\right) \sin \left(k d_{2}\right)}
$$

where $Z_{1}$ is the face-sheet impedance, $Z_{2}$ is the septum impedance, $d_{1}$ is the face sheet backing space depth, $d_{2}$ is the septum backing cavity depth, and $d=d_{1}+d_{2}$. Other models based on Helmholtz resonator or mass-spring-system analogy have been formulated in the context of broadband impedance eduction methods and to model the boundary conditions of lined ducts in the time domain [28, 30, 31, 32].

\subsection{Impedance model of the electroacoustic absorber}

Figure 2 (a) gives a schematic representation of an electrodynamic loudspeaker with a pressure-feedback control loop driven by a current source. Assuming that the diaphragm diameter is small compared to the wavelength in the frequency range of interest, the governing equation of the diaphragm and coil assembly can be expressed as [24, 25, 26]

$$
S_{d} p=\left(\mathrm{j} \omega M_{m s}+R_{m s}+\frac{1}{\mathrm{j} \omega C_{m s}}\right) v+B l i
$$

where $p$ is the total complex pressure at the diaphragm, $v$ is the velocity of the diaphragm, $i$ is the electrical current flowing through the coil, $S_{d}$ is the effective piston area of the diaphragm, $M_{m s}$ and $R_{m s}$ are the effective mass and mechanical resistance of the moving bodies, respectively, and $C_{m s}$ is the equivalent mechanical compliance of the suspension; $B l i$ is the Laplace force resulting from the magnetic field action on a free moving charge (current), where $B$ is the magnetic flux density and $l$ is the total length of the conductor. Since the loudspeaker is mounted on a box of volume $V_{b}$, the total mechanical compliance $C_{m c}=C_{m s}\left(1+\rho c^{2} S_{d}^{2} C_{m s} / V_{b}\right)^{-1}$, where $\rho=1.2 \mathrm{~kg} \mathrm{~m}^{-3}$ is the density of air and $c=343 \mathrm{~m} \mathrm{~s}^{-1}$ is the speed of sound in air, is substituted for $C_{m s}$ in Eq. (4).

Substituting $i=0$ into Eq. (4), the specific acoustic impedance of the loudspeaker diaphragm (in open circuit) takes the form of a mass-spring-damper system

$$
Z_{s}(\omega)=\frac{p}{v}=\frac{R_{m s}}{S_{d}}+\mathrm{j} \omega \frac{M_{m s}}{S_{d}}+\frac{1}{\mathrm{j} \omega S_{d} C_{m c}}
$$

where it is assumed that $Z_{s}$ is independent of the direction of incident sound, i.e. the diaphragm is a locally reacting surface. In order to achieve an actively tunable SDOF system, the target specific acoustic impedance can be formulated from Eq. (5) as

$$
Z_{s t}(\omega)=R_{s t}+\mathrm{j}\left(\mu_{1} \frac{\omega M_{m s}}{S_{d}}-\frac{\mu_{2}}{\omega S_{d} C_{m c}}\right)
$$

where $R_{s t}, \mu_{1}$ and $\mu_{2}$ are design parameters used to assign a desired resistance, mass and compliance at the loudspeaker diaphragm, respectively.

From continuity considerations at the boundary surface of the electroacoustic liner, the normal acoustic velocity $v_{n}$ is related to the diaphragm velocity $v$ over the effective piston area $S_{d}$ by

$$
v_{n}=\sigma v
$$


where $\sigma=S_{d} / S$ is the fractional effective area of a unit cell of surface $S$, as shown in Fig. 22(b). Substituting Eq. (7) into Eq. (6), the non-dimensional specific acoustic resistance and reactance in Eq. (1) can be rewritten as

$$
\theta=\frac{R_{s t}}{\rho c \sigma} \quad \text { and } \quad \chi=\frac{1}{\rho c \sigma}\left(\mu_{1} \frac{\omega M_{m s}}{S_{d}}-\frac{\mu_{2}}{\omega S_{d} C_{m c}}\right) .
$$

Equation (8) gives the relationship between the macroscopic parameters of the liner $(\theta$ and $\chi$ ), the physical parameters of the loudspeaker, and the design parameters used to meet the desired acoustic specifications.

\subsection{Electroacoustic transfer function}

For a current-driven loudspeaker, the control law by which a conventional electrodynamic loudspeaker is turned into an electroacoustic absorber can be derived by substituting Eq. (6) into Eq. (4) as

$$
i=\frac{S_{d}}{B l}\left(1-\frac{(\mathrm{j} \omega)^{2} M_{m s}+\mathrm{j} \omega R_{m s}+\frac{1}{C_{m c}}}{(\mathrm{j} \omega)^{2} \mu_{1} M_{m s}+\mathrm{j} \omega S_{d} R_{s t}+\frac{\mu_{2}}{C_{m c}}}\right) p
$$

and after some manipulations, the corresponding electroacoustic transfer function can be expressed as a second-order biquadratic transfer function [33]

$$
H(s)=K \frac{s^{2}+\frac{\omega_{z}}{Q_{z}} s+\omega_{z}^{2}}{s^{2}+\frac{\omega_{p}}{Q_{p}} s+\omega_{p}^{2}}
$$

where $s=\mathrm{j} \omega$ denotes the Laplace variable. The gain constant, characteristic frequency and quality factor of the numerator of Eq. (10) are given by

$$
K=\left(1-\frac{1}{\mu_{1}}\right) \frac{S_{d}}{B l}, \quad \omega_{z}=\sqrt{\frac{\mu_{2}-1}{\mu_{1}-1}} \frac{1}{\sqrt{M_{m s} C_{m c}}}, \quad Q_{z}=\frac{\left(\mu_{1}-1\right) M_{m s}}{S_{d} R_{s t}-R_{m s}} \omega_{z},
$$

and for the denominator in Eq. [10, the characteristic frequency and quality factor are

$$
\omega_{p}=\sqrt{\frac{\mu_{2}}{\mu_{1}}} \frac{1}{\sqrt{M_{m s} C_{m c}}}, \quad Q_{p}=\frac{\sqrt{\mu_{1} \mu_{2}}}{S_{d} R_{s t}} \sqrt{\frac{M_{m s}}{C_{m c}}} .
$$

Equation (10) corresponds to a tunable equalization filter which features adjustable gain at specified frequencies while leaving the remainder of the frequency response unchanged. The characteristics of Eq. (10) is completely determined by the five parameters $K, \omega_{z}, Q_{z}, \omega_{p}$ and $Q_{p}$. This permits simple frequency response adjustment by changing the values of the design parameters $\mu_{1}, \mu_{2}$, and $R_{s t}$, as indicated in Eqs. (11) and (12). Such a resonant filter can therefore be used to strengthen (boost) or attenuate (cut) the energy of specific frequency bands around the characteristic frequency. This basically involves (1) selecting the center frequency, (2) setting the $Q$ value which determines the sharpness of the bandwidth, and (3) adjusting the gain constant which determines how much those frequencies are boosted or cut relative to frequencies much above or below the selected center frequency. Table 1 examines the characteristics of the transfer function $H$ in Eq. $(10)$ as a function of the design parameters $\mu_{1}, \mu_{2}$ and $R_{s t}$.

As shown in Tab. 1. $H$ is a second-order band-reject transfer function when the design parameters are such that $\mu_{2}=\mu_{1}<S_{d} R_{s t} / R_{m s}$. In this case, Eq. $[10$ is a filter that attenuates a band of frequencies around the natural frequency of the loudspeaker while passing others unaltered. For $\mu_{2}=\mu_{1}$ and $R_{s t}=R_{m s} / S_{d}, H$ becomes a notch filter and we have almost infinite attenuation at $\omega_{z}=\omega_{p}$. For $\mu_{2}=\mu_{1}>S_{d} R_{s t} / R_{m s}$, on the other hand, $H$ is a second-order band-boost transfer function. Then, Eq. (10) is a peak filter applying a known gain that depends on the design parameters in the passband centered on $\omega_{z}=\omega_{p}$, while leaving all frequencies outside the passband unaltered. The transfer function $H$ degenerates into an all-pass filter for $\mu_{1}=\mu_{2}=S_{d} R_{s t} / R_{m s}$. For $\mu_{1}>\mu_{2}, H$ is a low-boost filter $\left(\omega_{p}<\omega_{z}\right)$ that can strengthen the energy of a specific frequency band below the natural frequency of the loudspeaker without filtering out the high frequencies, as a low-pass filter does. For $\mu_{1}<\mu_{2}$, on the other hand, $H$ is a high-boost filter that can strengthen the energy of a specific frequency band above the natural frequency of the loudspeaker. In both cases of lowor high-boost, the design parameters allows adjusting $Q_{p}$ and $Q_{z}$. Note that for $\mu_{1}=1, K=0$ and the liner is passive. When some of the open loop zeros, i.e. the roots of the numerator of Eq. (10), lie on the right-hand side of the complex s-plane, the system is non-minimum phase and is conditionally stable. Note also that $Q_{z}$ may be negative if $\mu_{1}>1$ and $\mu_{2}>1$, in which case $H$ is a non-minimum phase filter, while $Q_{p}$ must always be positive. 
Table 1: Main features of the electroacoustic transfer function 10 as a function of the design parameters $\mu_{1}, \mu_{2}$ and $R_{s t}$.

\begin{tabular}{lccc}
\hline Design settings & Characteristic frequency & Q factors & Filter function \\
\hline $0<\mu_{1}=\mu_{2}<1$ & $\omega_{z}=\omega_{p}$ & $\begin{array}{l}Q_{p}<Q_{z} \text { if } \mu_{1}<S_{d} R_{s t} / R_{m s} \\
Q_{p}>Q_{z} \text { if } \mu_{1}>S_{d} R_{s t} / R_{m s}\end{array}$ & $\begin{array}{c}\text { band-reject } \\
\text { band-boost }\end{array}$ \\
& $\omega_{z}=\omega_{p}$ & $Q_{p}=Q_{z}=\frac{1}{R_{m s}} \sqrt{\frac{M_{m s}}{C_{m c}}}$ & all-pass \\
$\mu_{1}=\mu_{2}=\frac{S_{d} R_{s t}}{R_{m s}}$ & $\omega_{z}>\omega_{p}$ & $\begin{array}{l}Q_{p}<Q_{z} \text { if } \mu_{2}<S_{d} R_{s t} / R_{m s} \\
Q_{p}>Q_{z} \text { if } \mu_{2}>S_{d} R_{s t} / R_{m s}\end{array}$ & low-boost \\
$0<\mu_{2}<\mu_{1}<1$ & & $Q_{p}>Q_{z}$ if $\mu_{1}>S_{d} R_{s t} / R_{m s}$ & high-boost \\
& $\omega_{z}<\omega_{p}$ & $Q_{p}<Q_{z}$ if $\mu_{1}<S_{d} R_{s t} / R_{m s}$ & \\
$0<\mu_{1}<\mu_{2}<1$ & & & \\
\end{tabular}

\section{Wave propagation in a flow duct}

This section presents the flow duct model used to study the performance of the electroacoustic liner flush mounted in the duct wall. It describes a rigid-walled duct of rectangular cross-section in the presence of a uniform mean flow and a multimodal excitation.

\subsection{Mathematical formulation of the problem}

Figure 3 illustrates the waveguide and coordinate system considered in this study ${ }^{1}$ The acoustic medium is a frictionless, homogenous (ideal) fluid of mass density $\rho$ subject to a steady axial flow with mean velocity $u_{0}$ that is assumed to be uniform over the cross section. The processes associated with wave motion are isentropic and fluctuating pressure amplitudes satisfy the linearized wave equation. Considering harmonic time dependence of the form $e^{j \omega t}$, the equations of the system described in Fig. 3 can be formulated in the frequency domain as follows:

- The convected 3D Helmholtz equation in the computational domain can be expressed in terms of the acoustic pressure $p$ as [3, 34]

$$
\left(1-M^{2}\right) \frac{\partial^{2} p}{\partial x^{2}}+2 \mathrm{j} k M \frac{\partial p}{\partial x}+\frac{\partial^{2} p}{\partial y^{2}}+\frac{\partial^{2} p}{\partial z^{2}}+k^{2} p=0
$$

where $M=u_{0} / c$ is the flow Mach number, $k=\omega / c$ is the wavenumber, $\omega$ is the angular frequency (in rad/s), and $c$ is the speed of sound in air (in $\mathrm{m} / \mathrm{s}$ ).

- For rigid-walled parts, the boundary conditions are given by

$$
\frac{\partial p}{\partial y}=0 \quad \text { at } \quad y=0, L_{y} \quad \text { and } \quad \frac{\partial p}{\partial z}=0 \quad \text { at } \quad z=0, L_{z}
$$

where $L_{y}$ and $L_{z}$ are the cross-sectional dimensions in the direction $y$ and $z$, respectively.

- For treated wall parts defined by any impedance model $Z$ corresponding to locally reacting liner or to the controlled electroacoustic absorber impedance given by Eq. (5), the Robin boundary conditions in the absence of flow are extended to a subsonic flow at Mach $M=u_{0} / c$ using the continuity of the acoustic normal displacement and acoustic pressure over an assumed infinite thin shear layer as [35, 36]

$$
\frac{\partial p}{\partial z}=\frac{1}{\mathrm{j} \omega Z}\left(\mathrm{j} \omega-M \frac{\partial}{\partial x}\right)^{2} p
$$

\footnotetext{
${ }^{1}$ Note that the coordinate variable ' $z$ ' must not be confused with the non-dimensional specific acoustic impedance in Eq 11 .
} 


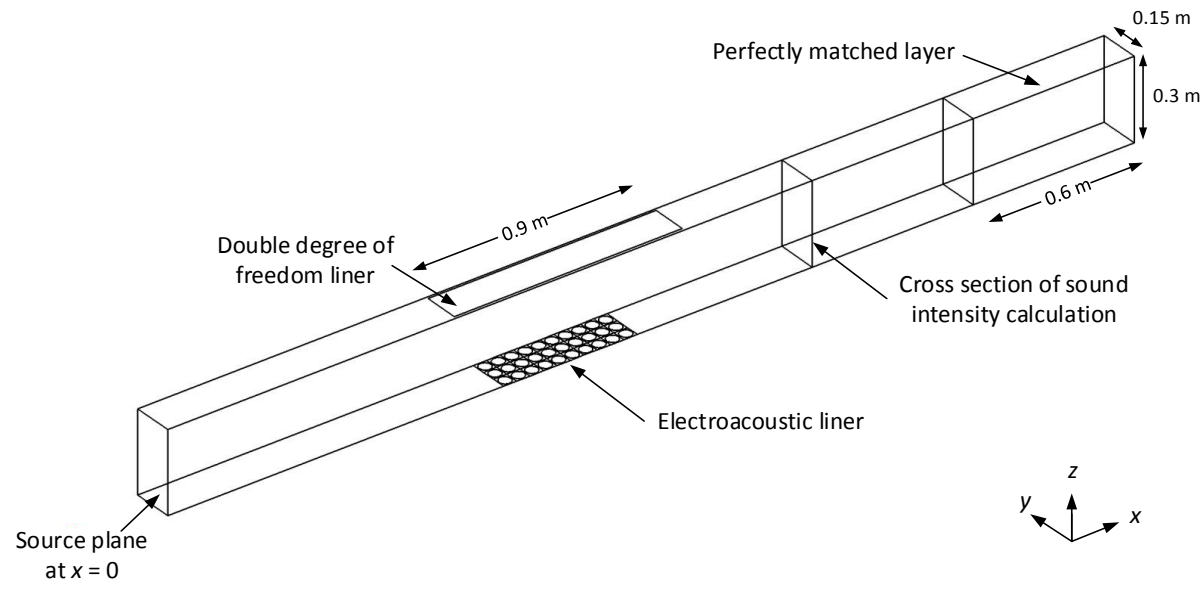

Figure 3: Geometry of the rectangular duct used in the numerical investigation.

- The boundary condition in the source plane located at $x=0$ (see Fig. 3 ) is a second-order radiation boundary condition defined in the frequency domain as [37]

$$
\frac{\partial}{\partial x}\left(p-p_{i}\right)+\mathrm{j} k\left(p-p_{i}\right)+\frac{\mathrm{j}}{2 k}\left(\frac{\partial^{2}}{\partial y^{2}}+\frac{\partial^{2}}{\partial z^{2}}\right)\left(p-p_{i}\right)=0,
$$

where the incident pressure field is a superposition of plane waves

$$
p_{i}(0, y, z)=\sum_{m} \sum_{n} A_{m n} \psi_{m n}(y, z),
$$

which is solution of the convected homogeneous Helmholtz equation. This non-reflecting boundary condition provides a well-posed problem in the finite computational domain and ensures that very little spurious wave reflection occurs from the assumed source distribution. To achieve an exact non-reflecting boundary condition, the Dirichlet-to-Neumann condition should be used instead. The modal amplitudes $A_{m n}$ and eigenfunctions $\psi_{m n}$ in Eq. (17) will be described in Section 3.3

- The boundary condition at the duct termination (right-hand side of Fig. 3) corresponds to a fully anechoic termination. In the numerical implementation described in Section 4.1, a perfectly matched layer (PML) is used to model this condition.

\subsection{Insertion loss}

After calculating the sound pressure in the computational domain, the insertion loss (IL) can be post-processed as described in this section. In the particular case of a duct with a uniform mean flow, the average intensity along the duct axis can be formulated as

$$
I_{x}=\frac{1}{2 \rho c} \operatorname{Re}\left[(p+M w)(w+M p)^{*}\right],
$$

where $p$ is the complex sound pressure and $w=\rho c v_{x}$ is an auxiliary variable that is related to the complex acoustic velocity $v_{x}$ along the duct axis. More details on the derivation of Eq. 18 ) are given in Appendix A.

The acoustic attenuation due to the electroacoustic lining is evaluated from the average intensity over a duct cross-section located downstream of the treated section. The IL is obtained according to

$$
\mathrm{IL}=10 \log _{10} \frac{I_{x 1}}{I_{x 2}}
$$

where the subscript 1 refers to the rigid-walled duct including the DDOF liner and subscript 2 refers to the rigid-walled duct including the DDOF liner and the electroacoustic liner, as shown in Fig. 3 . 


\subsection{Decomposition of the acoustic pressure field}

In this section, the decomposition of the acoustic pressure field is presented to provide the expression of sound excitation. In the duct section upstream of the treatment, the general solution of the boundary value problem given by Eqs. (13) and (14) can be obtained by separation of variables, and the acoustic pressure can be expressed in terms of rigid duct modes as [38]

$$
p(x, y, z)=\sum_{m=0}^{\infty} \sum_{n=0}^{\infty} p_{m n}(x) \psi_{m n}(y, z),
$$

where $p_{m n}$ are modal coefficients and, for a duct with rectangular cross-section, the normalized eigenfunctions are [39]

$$
\psi_{m n}(y, z)=\sqrt{\left(2-\delta_{0 m}\right)\left(2-\delta_{0 n}\right)} \cos \left(\frac{m \pi y}{L_{y}}\right) \cos \left(\frac{n \pi z}{L_{z}}\right) .
$$

where $\delta_{0 m}$ is the Kronecker delta function.

From Eq. (17), the incident pressure field in the duct section upstream of the treatment is, for a particular mode $(m, n)$, given by

$$
p_{m n}(x, y, z)=A_{m n} \psi_{m n}(y, z) e^{-\mathrm{j} k_{x m n} x},
$$

9 and using the linearized momentum equation, the modal acoustic velocity along the $x$-axis is [40]

$$
v_{x m n}(x, y, z)=\frac{1}{\rho c} \frac{\gamma_{m n}-M}{1-M \gamma_{m n}} A_{m n} \psi_{m n}(y, z) e^{-\mathrm{j} k_{x m n} x} .
$$

Substituting Eq. (21) in Eq. (13) gives the axial wavenumber [41]

$$
k_{x m n}=\frac{\gamma_{m n}-M}{1-M^{2}} k,
$$

where

$$
\gamma_{m n}=\left[1-\frac{1}{k^{2}}\left(\left(m \pi / L_{y}\right)^{2}+\left(n \pi / L_{z}\right)^{2}\right)\left(1-M^{2}\right)\right]^{1 / 2} .
$$

The condition for propagation of a given mode $(m, n)$ is that $k_{x m n}$ is real, i.e. $\gamma_{m n} \geqslant 0$, and the corresponding cut-on frequencies are

$$
\omega_{m n}=c \sqrt{\left(\left(m \pi / L_{y}\right)^{2}+\left(n \pi / L_{z}\right)^{2}\right)\left(1-M^{2}\right)} .
$$

When the liners are considered in the duct, as the eigenfunctions $\psi_{m n}$ form a complete basis, the pressure and axial velocity can be written in the equivalent matrix form

$$
p(x, y, z)=\boldsymbol{\Psi}^{T} \mathbf{p} \quad \text { and } \quad v_{x}(x, y, z)=\boldsymbol{\Psi}^{T} \mathbf{v},
$$

where $\mathbf{p}$ and $\mathbf{v}$ are vectors of modal coefficients, respectively, and $\boldsymbol{\Psi}$ is a vector of mode shape functions.

Without prior knowledge on source distribution or modal content in the experimental wind tunnel, we assumed that the total sound power is equally distributed among all propagating modes. The calculation of the modal amplitudes corresponding to a constant modal sound power model [41, 42] is given below. The total sound power may be obtained by the integration of the average intensity $(18)$ over the duct cross-section as

$$
\Pi=\frac{1}{2 \rho c} \int_{0}^{L_{y}} \int_{0}^{L_{z}} \operatorname{Re}\left[(p+M w)(w+M p)^{*}\right] \mathrm{d} y \mathrm{~d} z .
$$

From Eqs. (21 24) and (27), it can be shown that the acoustic power carried by a particular mode above cut-off in a uniform mean flow can be expressed as [40, 41]

$$
\Pi_{m n}=\left(2-\delta_{0 m}\right)\left(2-\delta_{0 n}\right) L_{y} L_{z} \frac{\left|A_{m n}\right|^{2}}{2 \rho c} \gamma_{m n}\left(\frac{1-M^{2}}{1-M \gamma_{m n}}\right)^{2} .
$$


In the case of the plane wave ( $m=n=0)$, we have $\delta_{0 m}=\delta_{0 n}=1$ and $\gamma_{m n}=1$, and it follows that the total sound power transported by the duct is

$$
\Pi=L_{y} L_{z} \frac{A_{00}^{2}}{2 \rho c}(1+M)^{2} .
$$

Assuming now that the total sound power is equally distributed over $N_{m}$ propagating modes, i.e. such that $\Pi_{m n}=\Pi / N_{m}$, the corresponding modal amplitudes can be expressed in terms of the plane wave amplitude $A_{00}$ as

$$
\left|A_{m n}\right|^{2}=\frac{1}{\left(2-\delta_{0 m}\right)\left(2-\delta_{0 n}\right)} \frac{1}{\gamma_{m n}}\left(\frac{1-M \gamma_{m n}}{1-M}\right)^{2} \frac{A_{00}^{2}}{N_{m}} .
$$

Equation 30 is used to define the sound excitation in Section 4.1 .

\section{Results}

This section provides numerical and experimental results that show the performance of the active electroacoustic liner described in Section 2, both in the absence and presence of flow. Without losing generality, an electroacoustic liner comprising an array of $3 \times 10$ loudspeakers arranged to fit the test section area of the duct flow facility is considered.

\subsection{Numerical investigation}

The convected 3D Helmholtz equation (13) with boundary conditions in Eqs. (14) and (15) is solved in the frequency domain using the finite element method (FEM). The problem is well posed and the discontinuity in the boundary condition and implementation of Robin boundary conditions $(15)$ is conform with the commonly used weak formulation. The geometry and coordinates of the studied flow duct is illustrated in Fig. 3 The multimodal excitation is generated in the source plane $(x=0)$ as a combination of incident plane waves, the amplitudes of which are defined from Eq. (30). The non-reflecting boundary condition is simulated at the duct termination using a PML. The cut-on frequencies derived from Eq. 25] are given in Tab. 2 for the flow duct under consideration (cross-sectional area $0.3 \times 0.15 \mathrm{~m}^{2}$ ). Note that this frequency domain simulation allow studying the steady state behaviour of the active liner only and does not take into account transient state.

Table 2: Cut-on frequencies up to $2 \mathrm{kHz}$ for the flow duct considered in this study.

\begin{tabular}{lcc}
\hline $\begin{array}{l}\text { Mode } \\
(m, n)\end{array}$ & No flow case $(M=0)$ & Flow case $(M=0.15)$ \\
\hline$(0,1)$ & $\omega_{m n} / 2 \pi$ & $\omega_{m n} / 2 \pi$ \\
$(0,2)$ & $1133 \mathrm{~Hz}$ & $560 \mathrm{~Hz}$ \\
$(0,3)$ & $1700 \mathrm{~Hz}$ & $1121 \mathrm{~Hz}$ \\
$(1,0)$ & $1133 \mathrm{~Hz}$ & $1681 \mathrm{~Hz}$ \\
$(1,1)$ & $1267 \mathrm{~Hz}$ & $1121 \mathrm{~Hz}$ \\
$(1,2)$ & $1602 \mathrm{~Hz}$ & $1253 \mathrm{~Hz}$ \\
$(1,3)$ & $2043 \mathrm{~Hz}$ & $1585 \mathrm{~Hz}$ \\
\hline
\end{tabular}

In the software platform the loudspeaker diaphragms are modeled as vibrating pistons according to Eq. (4). The control law given in Eq. (9) is implemented as an ordinary differential equation. Noticeable differences were observed 2 between loudspeakers during the experimental determination of their physical parameters. For the sake of simplicity, an average value calculated from the measurements was used in the model to describe each of the electromechanical parameters of the loudspeakers. To be consistent with the experimental investigation discussed below, a $0.9 \mathrm{~m}$ length DDOF liner is used in the opposite wall, as shown in Fig. 3. It is implemented in the FEM software platform using an impedance boundary condition. In our case, the DDOF liner includes a micro-perforated septum and is optimised for downstream propagation at $M=0.5$. The corresponding impedance model $Z_{\mathrm{ddof}}$ has been numerically evaluated 
through aeroacoustic simulations that provide a more precise model of the liner under grazing flow. Details are not provided here, however, readers who want to implement the model to validate the efficiency of the electroacoustic liner may use a model of the DDOF liner corresponding to Eq. (3) without loss of generality. A sound hard boundary condition is used elsewhere.

\subsection{Computed results}

A parametric study was carried out to evaluate the influence of the design parameters $R_{s t}, \mu_{1}$ and $\mu_{2}$ on the liner specific acoustic impedance. The results presented below correspond to a set of specific values and are intended to show the capability of the electroacoustic liner to achieve broadband performance. Table 3 summarizes the liner acoustic performance for the studied configurations. The comparison of the IL calculated and measured from the values of Tab. 3 is presented in Section 4.4 The determination of the optimal attenuation at specified frequencies with respect to the specific acoustic impedance of the liner is discussed in Section 4.5
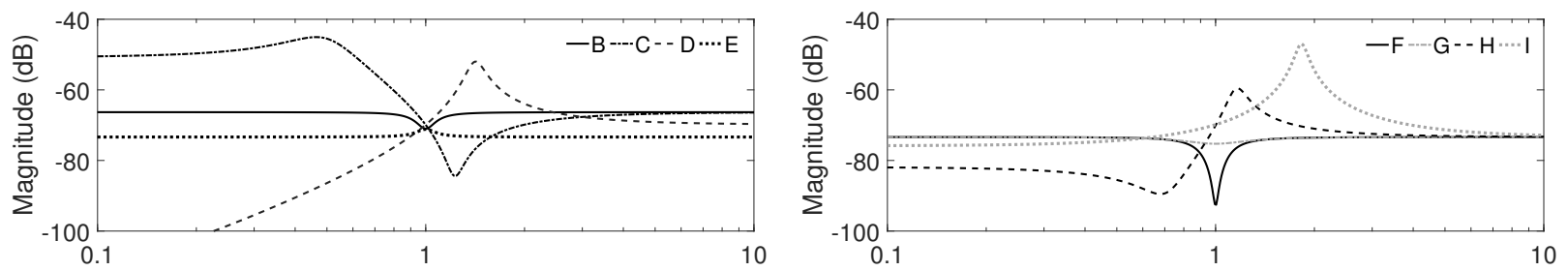

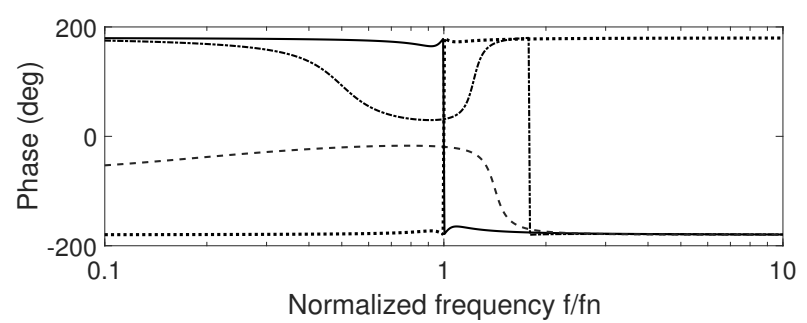

(a)

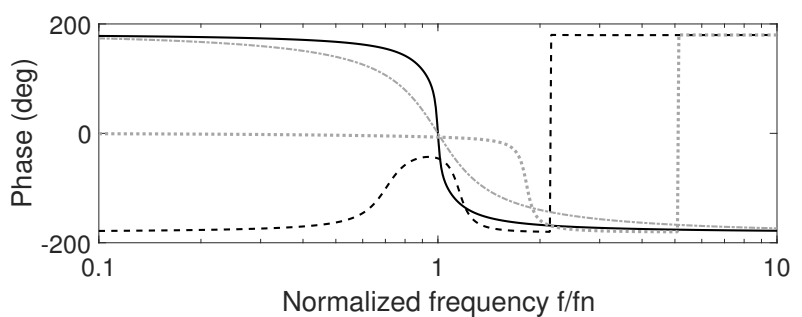

(b)

Figure 4: Bode plot of the transfer functions calculated from Eq. 10 for configurations B,C, D and E (a), and configurations F, G, H and I (b) (see Tab. 3 .

Figure 4 shows the Bode plot of the electroacoustic transfer functions of Eq. (10) for the configurations listed in Tab. 3 As discussed above, these transfer functions correspond to the control law by which the loudspeaker is driven from the sound pressure at its diaphragm. As shown in Fig. 4, different frequency response functions can be obtained depending on the design parameters. When $\mu_{1}=\mu_{2}<S_{d} R_{s t} / R_{m s}$ as in cases B, F and G, the electroacoustic transfer function is a band-reject filter whose center frequency is the natural frequency of the loudspeaker, as indicated in Tab. 1 As can be seen in Fig. 4 (a) and (b), the Q factor of the resonant filter depends directly on the value of the design parameters. When $\mu_{1}=\mu_{2}>S_{d} R_{s t} / R_{m s}$ as in case E, on the other hand, Eq. [10] is a band-boost filter which presents a peak in the frequency response at the natural frequency of the loudspeaker. For $\mu_{1} \neq \mu_{2}$ in Eq. (9), the corresponding electroacoustic function is a low-boost filter if the ratio $\mu_{1} / \mu_{2}>1$ (see case $\mathrm{C}$ ), and a high-boost filter if the ratio $\mu_{1} / \mu_{2}<1$ (see cases D, H and I).

Figure 5 shows the non-dimensional specific acoustic impedance calculated from Eq. (6) for the studied configurations. This calculation result illustrates the specific acoustic impedance that is assigned to each diaphragm for the control settings listed in Table 3. As shown in Fig. 5) the design parameters used in the control law (9) make it possible to adjust the specific acoustic impedance of the loudspeakers diaphragm. By varying the ratio $\mu_{1} / \mu_{2}$ for a given value of $R_{s t}=\rho c / 2<R_{m s} / S_{d}$ (see cases B, C, D, E, H, and I), it can be observed that the reactance of the loudspeaker diaphragm is changed: the resonance frequency (where the non-dimensional specific acoustic reactance curve crosses zero) is shifted and the slope is decreased compared with the uncontrolled loudspeaker (case A). Conversely, increasing the value for $R_{s t}$ for a constant ratio $\mu_{1} / \mu_{2}$ (see cases E, F, and G in Fig. 5(b)) results in an increase of the diaphragm specific acoustic resistance without modifying its reactance. Not shown in this paper, decreasing the value for $R_{s t}$ causes a decreases of the diaphragm resistance while leaving the reactance unchanged accordingly. 

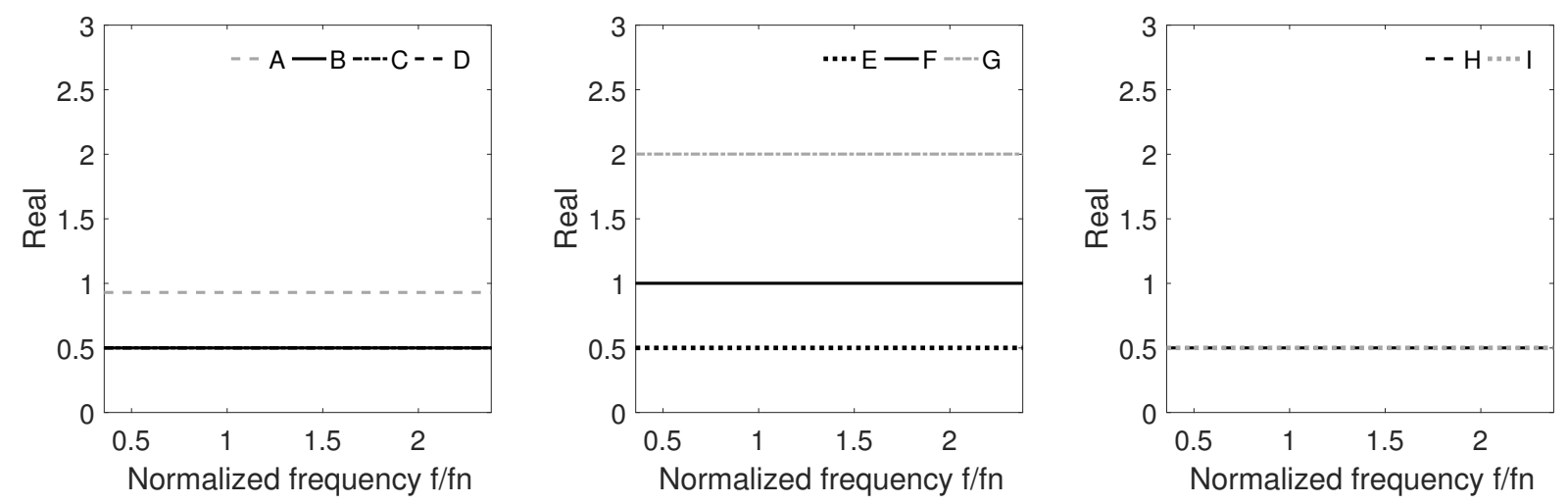

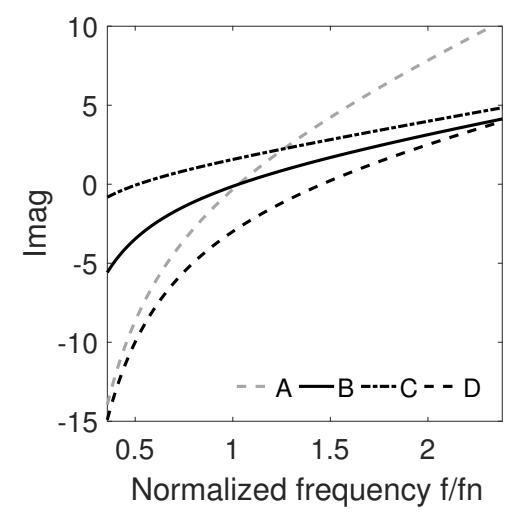

(a)

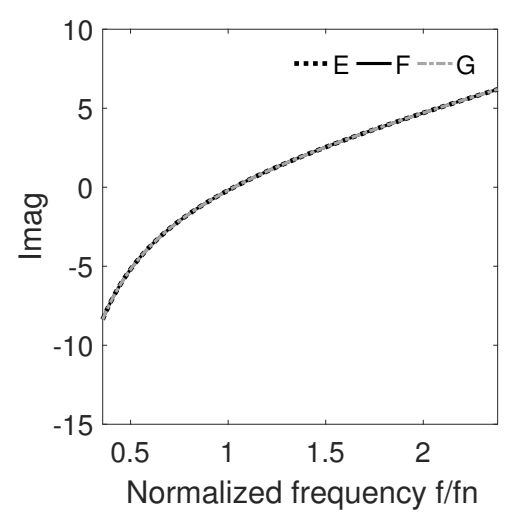

(b)

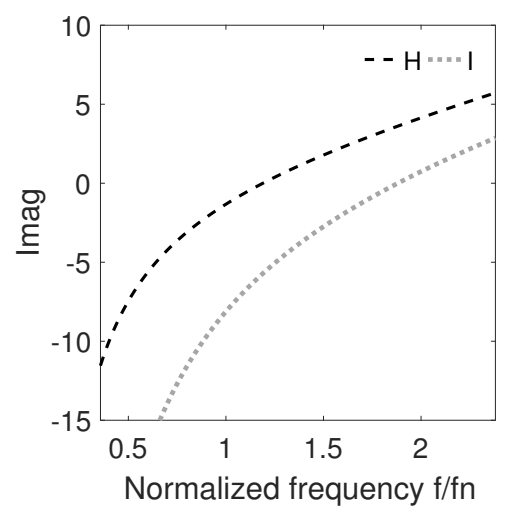

(c)

Figure 5: Real part and imaginary part of the non-dimensional specific acoustic impedance at the loudspeaker diaphragm calculated from Eq. 6 for configurations A,B,C and D (a), E,F, and G (b), and H and I (c) (see Tab. 3.

\subsection{Experimental setup}

Experiments were carried out to determine the performance of the electroacoustic liner prototype in terms of IL with and without airflow. The measurement were conducted in the flow duct facility of the Netherland Aerospace Center (NLR). As depicted in Fig. 6, the test section (length $1.05 \mathrm{~m}$, cross-sectional area $0.3 \times 0.15 \mathrm{~m}^{2}$ ) is flush-mounted into the wind tunnel that connects two reverberation chambers in which the sound pressure level (SPL) is measured to determine the acoustic attenuation provided by the treatment under grazing flow conditions [43]. The first duct cut-on frequency for $M=0$ is $567 \mathrm{~Hz}$ for the no-flow case, which implies a multimodal propagation for $f>567 \mathrm{~Hz}$. The IL was measured by comparing the change in SPL due to the insertion of the electroacoustic liner into the duct connecting the two reverberation chambers, with air flowing through the treated section to simulate engine conditions. Sound excitation is generated in the sending (source) reverberation chamber using acoustic drivers. Over the frequency range of interest, the acoustic field inside the two reverberation chambers are considered to be diffuse. A rotating microphone located in the downstream reverberation chamber was used to obtain a spatially averaged value of the acoustic pressure. The averaging time of the spectrum analyzer is selected to be greater than the rotating period of the microphone. The IL is obtained by first measuring the SPL $L_{p 1}$ in the reverberation chambers with the $0.9 \mathrm{~m}$ DDOF liner only, and then measuring the SPL $L_{p 2}$ with the liner under study and the DDOF liner opposite to it. The difference (in decibels) measured in the downstream (receiving) reverberation chamber give the attenuation of the treatment, i.e. $\mathrm{IL}=L_{p 1}-L_{p 2}$. This measured value takes into account source and termination effects. For experimental assessment, the sound pressure level of the excitation was around $114 \mathrm{~dB}$.

The electroacoustic liner prototype shown in Fig. 7(a) consists in an arrangement of 3 rows of 10 unit cells, each covering a surface area $S=0.05 \times 0.05=0.0025 \mathrm{~m}^{2}$. A unit cell includes a closed-box loudspeaker, four microphones distributed around the diaphragm and an electronic control board with microcontrollers. The average sound pressure 


\begin{tabular}{|c|c|c|c|c|c|c|c|c|c|}
\hline \multirow[b]{2}{*}{ Case } & \multicolumn{4}{|c|}{ Design parameters value } & \multicolumn{3}{|c|}{ Computed value } & \multicolumn{2}{|c|}{ Measured value } \\
\hline & $R_{s t}$ & $\mu_{1} / \mu_{2}$ & $\omega_{p} / \omega_{n}$ & $Q_{p}$ & $\omega_{\text {res }} / \omega_{n}$ & $Z_{s t} / \rho c$ & IL & $\omega_{\text {res }} / \omega_{n}$ & IL \\
\hline \multicolumn{10}{|c|}{ No flow $(M=0)$} \\
\hline A & no control & & & & 0.97 & $1.58-0.48 \mathrm{j}$ & $10.5 \mathrm{~dB}$ & 0.95 & $7.7 \mathrm{~dB}$ \\
\hline B & $\rho c / 2$ & 1 & 1 & 4.4 & 0.94 & $0.85-0.53 \mathrm{j}$ & $21.7 \mathrm{~dB}$ & 0.92 & $15.5 \mathrm{~dB}$ \\
\hline $\mathrm{C}$ & $\rho c / 2$ & 4 & 0.5 & 2.2 & 0.54 & $0.69-0.54 j$ & $6.6 \mathrm{~dB}$ & 0.5 & $7.5 \mathrm{~dB}$ \\
\hline $\mathrm{D}$ & $\rho c / 2$ & 0.5 & 1.41 & 7.8 & 1.3 & $0.95-1.15 \mathrm{j}$ & $13.2 \mathrm{~dB}$ & 1.27 & $10.2 \mathrm{~dB}$ \\
\hline \multicolumn{10}{|c|}{ Mean flow $(M=0.15)$} \\
\hline E & $\rho c / 2$ & 1 & 1 & 6.6 & 0.95 & $0.87-0.63 j$ & $20.4 \mathrm{~dB}$ & 0.95 & $14.9 \mathrm{~dB}$ \\
\hline $\mathrm{F}$ & $\rho c$ & 1 & 1 & 3.3 & 0.95 & $1.66-0.56 j$ & $10.2 \mathrm{~dB}$ & 0.95 & $10.0 \mathrm{~dB}$ \\
\hline G & $2 \rho c$ & 1 & 1 & 1.6 & 0.94 & $3.18-0.81 \mathrm{j}$ & $5.4 \mathrm{~dB}$ & 0.95 & $7.4 \mathrm{~dB}$ \\
\hline $\mathrm{H}$ & $\rho c / 2$ & 0.75 & 1.15 & 7.6 & 1.1 & $1.02-0.78 j$ & $15.4 \mathrm{~dB}$ & 1.09 & $10.7 \mathrm{~dB}$ \\
\hline I & $\rho c / 2$ & 0.3 & 1.83 & 12 & 1.72 & $1.11-1.60 \mathrm{j}$ & $5.0 \mathrm{~dB}$ & 1.70 & $5.8 \mathrm{~dB}$ \\
\hline
\end{tabular}

obtained from the four microphones is used as input to the controller. The loudspeakers have been specifically designed at the Centre de Transfert de Technologie du Mans (CTTM) to meet specified geometric and technological requirements. Not shown in this paper, the electronic control board can provide up to $250 \mathrm{~mA}$ per loudspeaker for a power supply voltage of $5 \mathrm{~V}$, a peak power rating of $1.25 \mathrm{~W}$. The total electrical power of the active liner in operation is between $12.5 \mathrm{~W}$ and $30 \mathrm{~W}$. Moreover, a protective grid is added in front of the electroacoustic liner so as to protect the diaphragm from the air flow. It simply consists of a thin perforated polycarbonate plate ( $2 \mathrm{~mm}$ thick, $1.8 \mathrm{~mm}$ hole diameter) with a large open area so as not to add additional acoustic resistance to the lining.

\subsection{Experimental validation}

Numerically computed and experimentally obtained acoustic performance comparisons are presented in Fig. 8 Numerical predictions of the sound attenuation are in good agreement with the measured data, both in the presence of flow and in the absence of flow. The differences observed between measurements and simulations are partly due to some variation in the loudspeakers electromechanical parameters, which can be up to $10 \%$ between any two loudspeakers. This dispersion causes the diaphragms to present different resonance frequencies and internal mechanical losses. In the experimental results presented above, these differences are not compensated in the control law. Moreover, the gain value $\mu_{1} / \mu_{2}$ can amplify the differences, as in cases C, D, H and I. In the model, however, the values of the parameters applied to the diaphragms correspond to the average of the values of the electromechanical parameter measured on all the loudspeakers. This explains why the performance is overestimated in the simulations. Note also that the load impedance of the reverberation chamber is not included in the simulation, which may also explain some of the observed discrepancies between calculated and measured data. As expected, the active electroacoustic liner prototype provides a very high level of IL in the low frequency range around the loudspeaker resonance frequency (see cases B and E). As shown in Fig. 8, the peak of maximum attenuation obtained experimentally for the configurations studied is about 15 $\mathrm{dB}$ at $0.94 \cdot f_{n}$, i.e. around the natural frequency of the loudspeakers. By adjusting the design parameters, furthermore, a good attenuation can be achieved in a wide frequency range, between $f_{n} / 2$ and almost $2 \cdot f_{n} \mathrm{~Hz}$ for the configurations studied in this paper. As expected by varying the ratio $\mu_{1} / \mu_{2}$, the peak of attenuation is shifted in a frequency range that is close to the center frequency of the corresponding electroacoustic filter, which is given by $\sqrt{\mu_{2} / \mu_{1}} \cdot f_{n}$ (see Tab. 3), where $f_{n}=1 /\left(2 \pi \sqrt{M_{m s} C_{m c}}\right)$ is the natural frequency of the loudspeaker. The moderate reduction obtained by control below and above the natural frequency of the loudspeaker, respectively $7.5 \mathrm{~dB}$ at $0.54 \cdot f_{n}$ for case $\mathrm{C}$ with a 


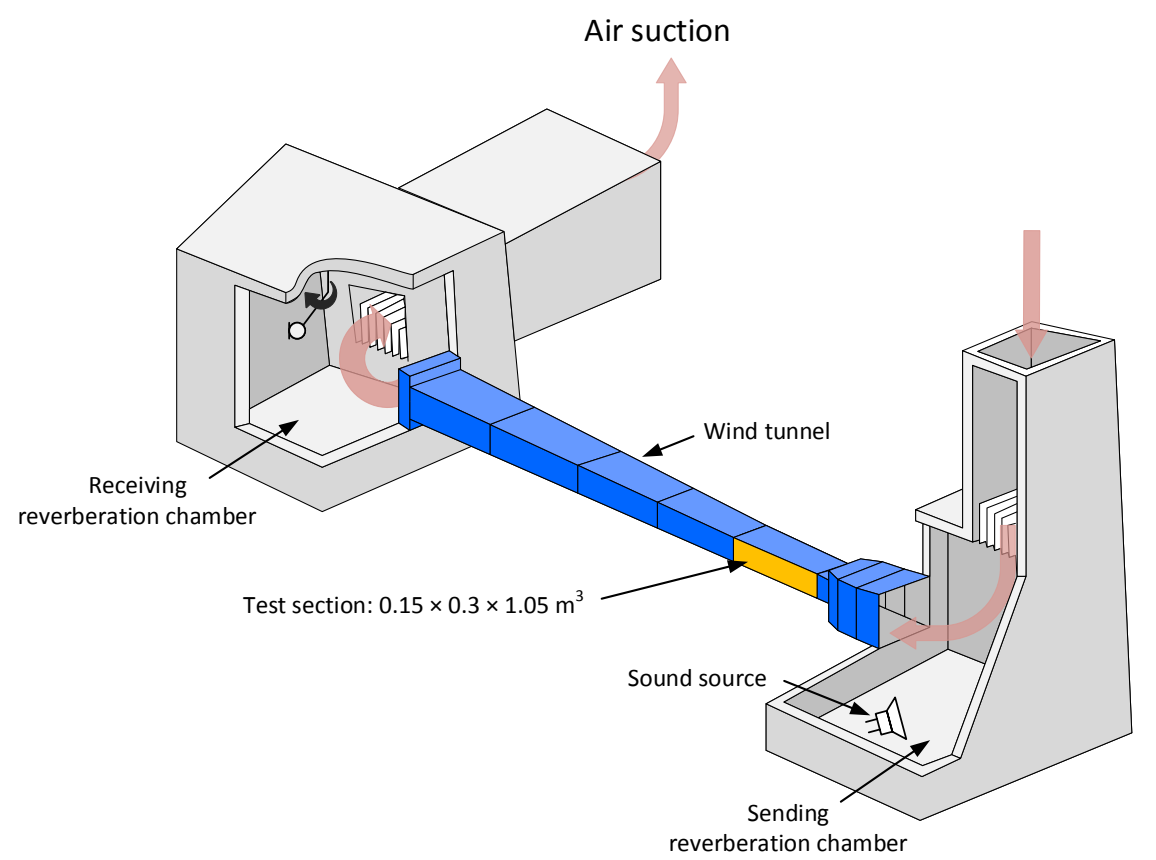

Figure 6: Acoustic flow duct facility used in the experimental assessment at NLR.

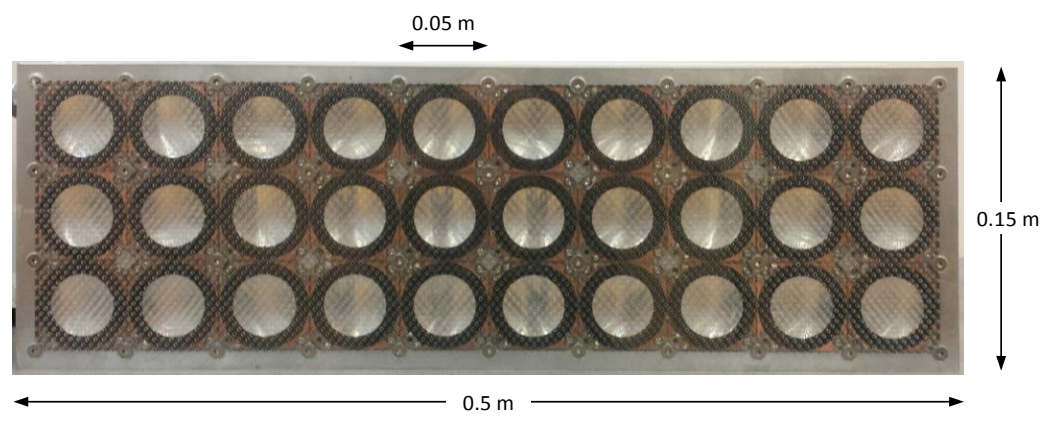

(a)

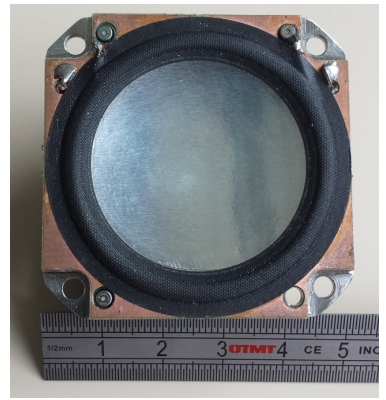

(b)

Figure 7: Pictures and dimensions of the electroacoustic liner prototype (a) and single control unit (b).

1 ratio $\mu_{1} / \mu_{2}=4$ and $5.8 \mathrm{~dB}$ at $1.72 \cdot f_{n}$ for case I with a ratio $\mu_{1} / \mu_{2}=0.3$, is consistent with the theory.

\section{4.5. Maximum achievable attenuation}

A second parametric study was performed afterwards to determine which specific acoustic impedance would lead to the maximum attenuation under the same experimental conditions. The corresponding values in terms of the design parameters can be derived from Eq. 8). Figure 9 shows the constant attenuation contours in $\mathrm{dB}$ with and without airflow computed as a function of the liner complex impedance at some specified frequencies (see Tab. 3). As shown in Fig. 9, the maximum IL level for the flow duct model and a multimodal excitation is frequency dependent. Overall, it is found that the optimal acoustic resistance and reactance both increase with frequency, with or without flow. As can be seen in Fig. 9(a), the maximum attenuation below the natural frequency of the loudspeaker is expected for a low resistance, i.e. $z \simeq 0.2-0.6 j$ at $f=0.54 \cdot f_{n} \mathrm{~Hz}$; the maximum IL is calculated at almost $45 \mathrm{~dB}$. Around the natural frequency of the loudspeaker the maximum IL reaches $35.4 \mathrm{~dB}$ for $z \simeq 0.5-0.55 \mathrm{j}$ at $f=0.94 \cdot f_{n} \mathrm{~Hz}$, and $17.1 \mathrm{~dB}$ for 


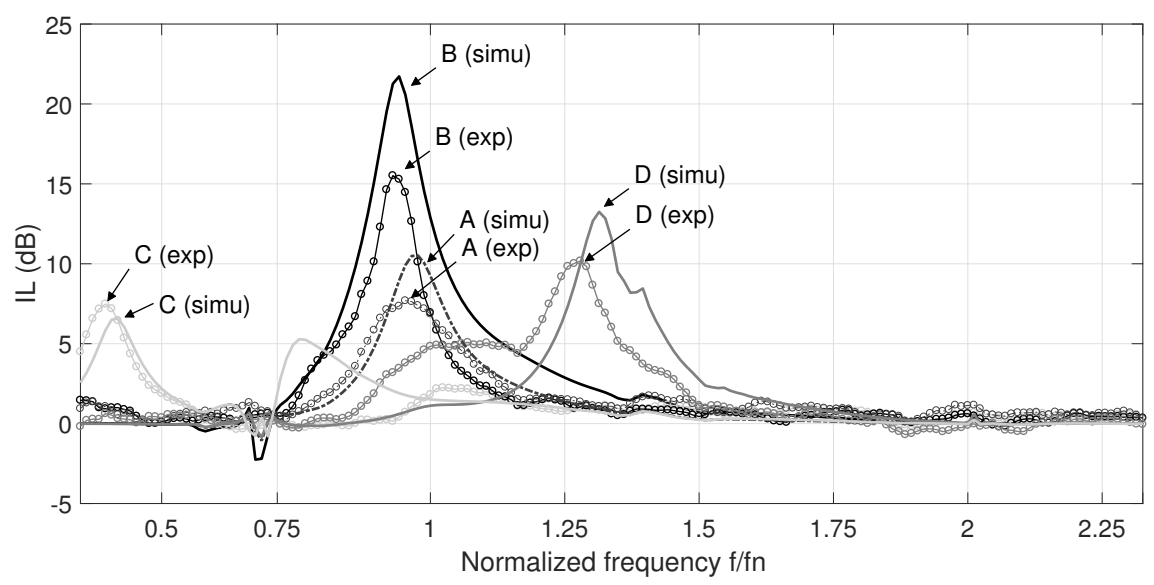

(a)

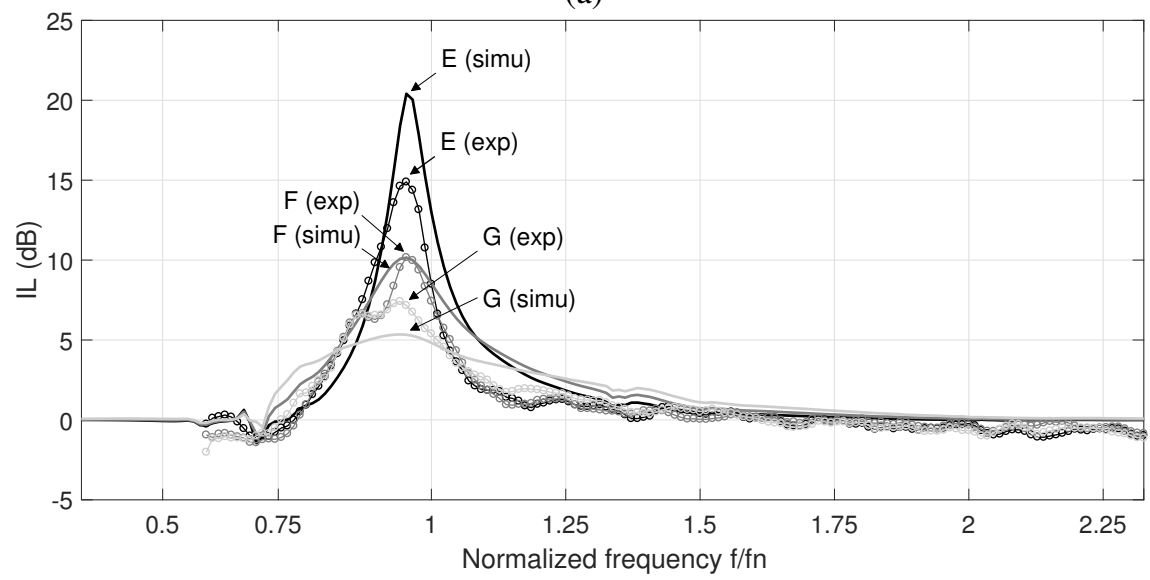

(b)

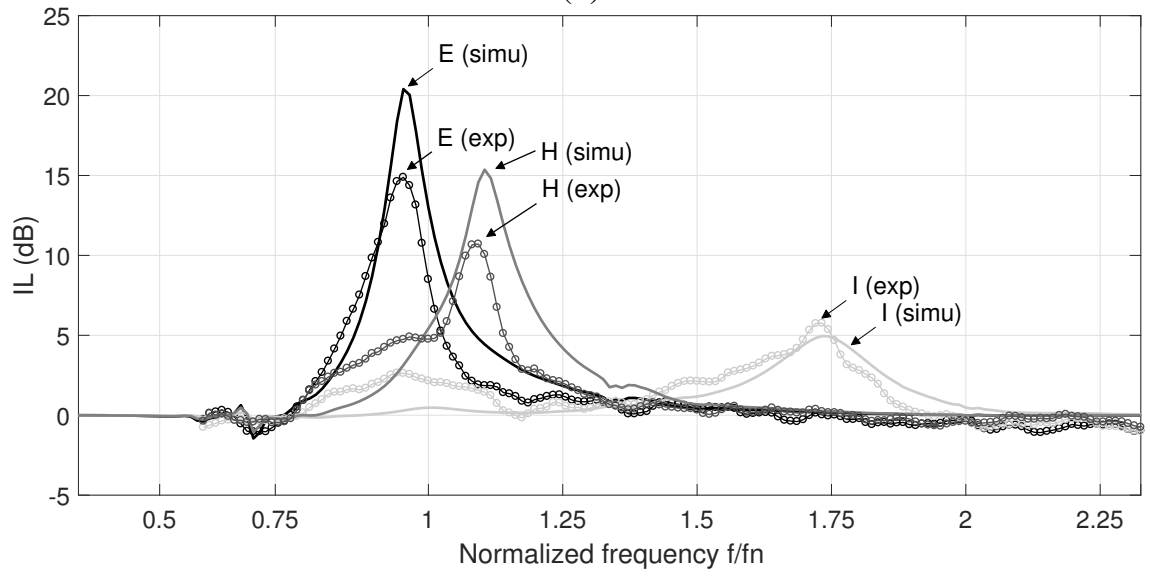

(c)

Figure 8: Comparisons of acoustic attenuation in terms of IL for the design parameters given in Tab. 3; (a) shows the IL obtained in the absence of mean flow $(M=0)$ for case A (passive electroacoustic liner), case B $\left(R_{s t}=\rho c / 2\right.$ and $\left.\mu_{1} / \mu_{2}=1\right)$, case $\mathrm{C}\left(R_{s t}=\rho c / 2\right.$ and $\left.\mu_{1} / \mu_{2}=4\right)$, and case $\mathrm{D}$ $\left(R_{s t}=\rho c / 2\right.$ and $\left.\mu_{1} / \mu_{2}=0.5\right)$; (b) and (c) show the IL obtained in the presence of low mach number flow $(M=0.15)$ for case $\mathrm{E}\left(R_{s t}=\rho c / 2\right.$ and $\left.\mu_{1} / \mu_{2}=1\right)$, cas F $\left(R_{s t}=\rho c\right.$ and $\left.\mu_{1} / \mu_{2}=1\right)$, case $\mathrm{G}\left(R_{s t}=2 \rho c\right.$ and $\left.\mu_{1} / \mu_{2}=1\right)$, case $\mathrm{H}\left(R_{s t}=\rho c / 2\right.$ and $\left.\mu_{1} / \mu_{2}=0.75\right)$ and case I $\left(R_{s t}=\rho c / 2\right.$ and $\left.\mu_{1} / \mu_{2}=0.3\right)$. Computed data are plotted in solid line and measured data are plotted in solid line with circle markers. 


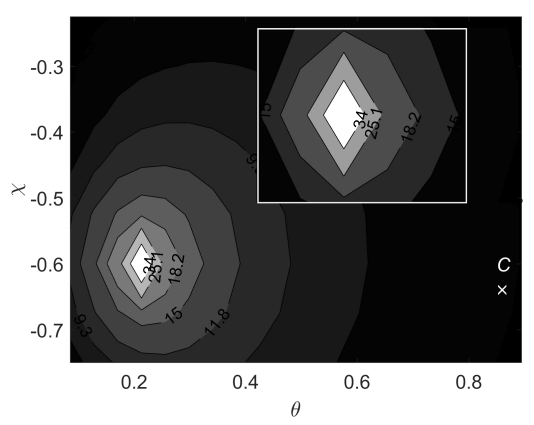

(a)

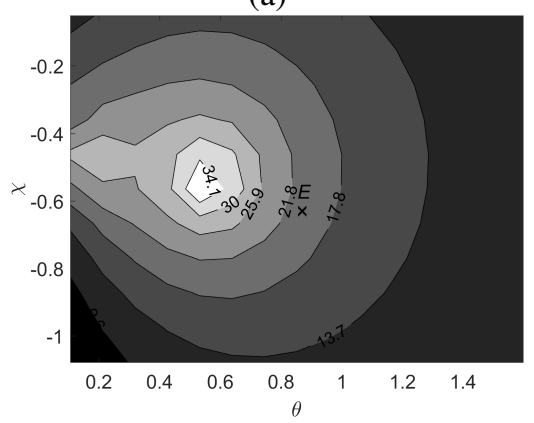

(d)

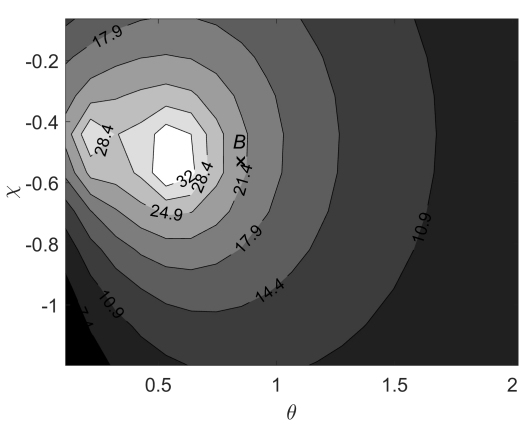

(b)

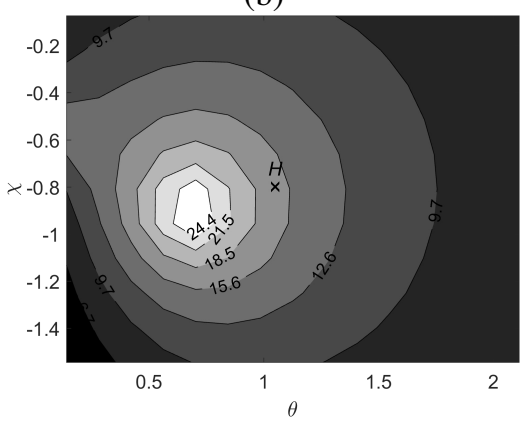

(e)

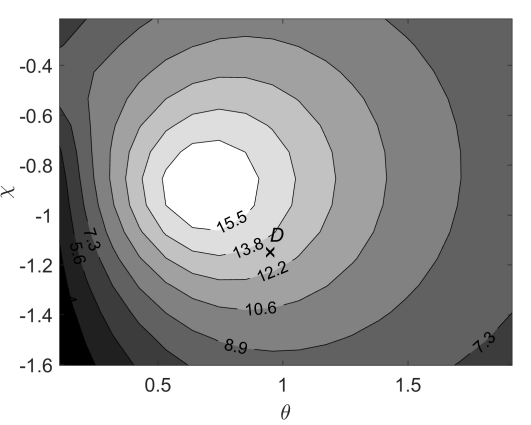

(c)

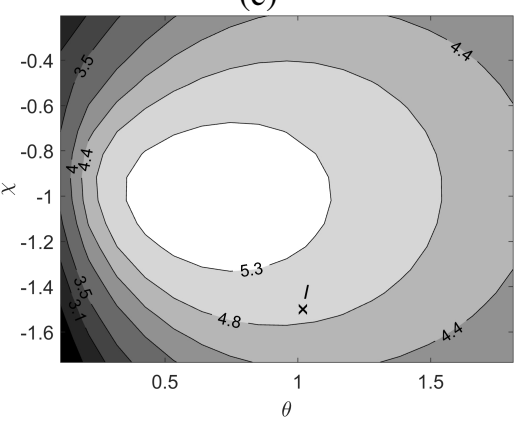

(f)

Figure 9: Constant IL contours (in $\mathrm{dB}$ ) calculated as a function of the non-dimensional specific acoustic resistance and reactance of the liner in the absence of flow, (a) at $f=0.54 \cdot f_{n} \mathrm{~Hz}$, (b) at $f=0.94 \cdot f_{n} \mathrm{~Hz}$, and (c) at $f=1.3 \cdot f_{n} \mathrm{~Hz}$, and with airflow, (d) at $f=0.95 \cdot f_{n} \mathrm{~Hz},(\mathrm{e})$ at $f=1.1 \cdot f_{n}$ $\mathrm{Hz}$, and (f) at $f=1.72 \cdot f_{n} \mathrm{~Hz}$. The configuration studied at those particular frequencies is plotted with marker ' $\times$ ' (see Tab. 3 .

$z \simeq 0.7-0.85 \mathrm{j}$ at $f=1.3 \cdot f_{n} \mathrm{~Hz}$. In the presence of flow, the maximum IL level is a bit higher compared to the no flow case and reaches $38.2 \mathrm{~dB}$ for a non-dimensional specific acoustic impedance $z \simeq 0.55-0.55 \mathrm{j}$ at $f=0.95 \cdot f_{n} \mathrm{~Hz}$; the maximum IL is of about $27.4 \mathrm{~dB} z \simeq 0.65-0.9 \mathrm{j}$ at $f=1.1 \cdot f_{n} \mathrm{~Hz}$, and $5.4 \mathrm{~dB}$ for $z \simeq 0.75-1 \mathrm{j}$ at $f=1.72 \cdot f_{n}$ Hz. As can be seen in Fig. 9, the lower the frequency, the smaller the optimal attenuation area in the complex $s$-plane. Maximum IL is much more sensitive to small variations in impedance in lower than in high frequencies.

\subsection{Discussion}

Experimental results confirm that the active lining concept allows to locally achieve a given acoustic impedance, which can be adjusted in real time to, for example, adapt to the speed of the engine. The wind tunnel tests were performed for a maximum flow velocity of $M=0.15$ to prevent flow-induced noise from overloading the microphones of the active liner, which corresponds to a sound pressure level around $114 \mathrm{~dB}$ close to the lining section. It is worth mentioning that noise in engine nacelles can typically reach $160 \mathrm{~dB}$ and flow velocity $M=0.6$. Of primary concern in real-world applications is therefore the capability of the active electroacoustic absorbers to interact with the fan noise and to withstand potentially high amplitude of pressure fluctuations within the engine nacelle environment. For operation in the low- and mid-frequency range, typically below $2 \mathrm{kHz}$, conventional electrodynamic loudspeakers are efficient and reliable, but they have a lightweight diaphragm that would be likely to damage from intense fluctuating pressures. Even though no damage to the loudspeaker diaphragms was found during the tests carried out under those conditions, the mechanical rigidity of the diaphragm and the accuracy of the lumped element model for harsh conditions should be both considered in future work. An alternative would be to use a piezo-electric transducer bonded to an aluminum diaphragm. This approach is more compact and lightweight and can be designed to have a high mechanical impedance, protecting it from high fluctuating pressure amplitudes. The drawback is that the high mechanical impedance would restrict vibration amplitude.

This active liner concept is based on a multi-channel decentralised control system. Each processor (or node) receives a single sensor signal (here the average pressure of the four microphone around the diaphragm) from which a single control signal is delivered to the loudspeaker driver. The decentralised control scheme allows for parallel computation 
of control variables but absence of communication between controllers may limit the achievable performance. The advantage of the decentralised architecture is, however, to be robust even if some of the control units are disabled. During the experiment in the wind tunnel, some control units were disabled without disturbing the other electroacoustic absorbers.

In contrast to ducted ventilation systems and mufflers where the small cross-section predominantly allows the propagation of plane waves, the transverse dimensions of high-bypass turbofan engine nacelle permit wave propagation of many higher-order modes. To approximate such a multimodal excitation, the simulations were carried out in the frequency domain by generating a constant modal sound intensity distribution [42] in the source plane at the duct inlet. In a flow duct facility such as that shown in Fig. 6, on the other hand, the phase angles of the propagation modes depend, among other things, on the space-time pattern of the fan noise source distribution. If source distributions with temporal and spatial incoherence are assumed, the phase angles vary randomly with space and time, as discussed by Doak [39]. In this study, modal contributions were applied in the source plane without taking into account temporal and spatial incoherence between them. To provide a more realistic excitation, it would be interesting to consider the effects of random spatial and temporal character of the source distribution in future work. In addition, time-domain simulation should be also considered to account for arbitrary base flow conditions and transient and non-linear propagation phenomena. In this regard, it would be valuable to verify that the equivalent time-domain impedance boundary condition is physically realizable, i.e. the impedance function must be causal, real, and passive [31].

\section{Conclusion}

This paper presents an active lining concept based on an arrangement of electroacoustic absorbers, consisting in feedback-controlled loudspeaker membranes in a decentralised scheme, and its application to duct modes damping. This electroacoustic liner was theoretically studied using a lumped element model and its ability to achieve a desired specific acoustic impedance in a flow duct was evaluated. The IL of the liner was calculated numerically for various design parameters and compared to measurements performed in a flow duct facility. The parametric study carried out by simulation showed the influence of the design parameters on the overall performance of the liner. This electroacoustic liner has an attenuation potential comparable to that of a conventional passive liner, but offers greater flexibility to achieve a given complex acoustic impedance in the low frequencies, which is moreover adaptive in real time. It is shown that the acoustic resistance and reactance of the liner can be tuned independently, which allows the duct modes to be attenuated over a bandwidth of two octaves around the resonance frequency of the loudspeakers. The level of peak attenuation and target frequency are, for a given duct geometry and flow condition, controlled by the diaphragm acoustic resistance $R_{s t}$ and reactance through the ratio $\mu_{2} / \mu_{1}$, respectively, and related to the percentage of effective area $\sigma$ of the liner. When the target frequency is below the natural frequency of the loudspeaker, the target resistance $R_{s t}$ decreases rapidly and may be much less than $\rho c$. In addition to offering an adaptive locally reacting impedance, one advantage of the multi-channel decentralised control system used to implement the liner is that it remains robust even if some of the control units are disabled. In future work, it is planned to combine the decentralised control scheme with a distributed control law [21] to improve performance.

\section{Acknowledgment}

This project has received funding from the European Union's Seventh Framework Programme for research technological development and demonstration under grant agreement no 604999. The authors would like to acknowledge Safran Nacelles and NLR for the support, and Rik Wijntjes for his contribution during the experiments. FEMTO-ST contribution received support from Labex ACTION progam (ANR-11-LABX-0001-01).

\section{Appendix A. Sound intensity in a uniform mean flow}

In the presence of flow $\mathbf{M}=\mathbf{u}_{0} / c$, where $\mathbf{u}_{0}$ is the steady-state flow velocity, the instantaneous sound intensity vector is defined as [40]

$$
\mathbf{I}_{i}(t)=p(t) \mathbf{v}(t)+\frac{\mathbf{M}}{\rho c} p^{2}(t)+\mathbf{M}(\mathbf{M} \cdot \mathbf{v}(t) p(t))+\rho c \mathbf{v}(t)(\mathbf{v}(t) \cdot \mathbf{M})
$$


where $p(t)$ and $\mathbf{v}(t)$ are the instantaneous sound pressure and acoustic velocity and $\mathbf{M}$ is the Mach number. The time-averaged active sound intensity vector is given by

$$
\mathbf{I}=\left\langle\mathbf{I}_{i}(t)\right\rangle=\frac{1}{2} \operatorname{Re}\left[p \mathbf{v}^{*}+\frac{\mathbf{M}}{\rho c} p p^{*}+\mathbf{M}\left(\mathbf{M} \cdot\left(p \mathbf{v}^{*}\right)\right)+\rho c \mathbf{v}\left(\mathbf{v}^{*} \cdot \mathbf{M}\right)\right],
$$

where * denotes the complex conjugate.

In the particular case of a duct with a uniform mean flow such that $\mathbf{M}$ is parallel to the duct axis, the component of the time-averaged active sound intensity vector along the duct axis can be expressed as:

$$
I_{x}=\frac{1}{2} \operatorname{Re}\left[p v_{x}^{*}\left(1+M^{2}\right)+M\left(\frac{p p^{*}}{\rho c}+\rho c v_{x} v_{x}^{*},\right)\right] .
$$

where $p$ and $v_{x}$ are the complex sound pressure and axial acoustic velocity and $M$ is the Mach number. By introducing the auxiliary variable $w=\rho c v_{x}$, Eq. A.3 can be rewritten as Eq. [18.

\section{References}

[1] P.M. Morse and K.U. Ingard, Theoretical acoustics, McGraw-Hill, New York (1968).

[2] A.W. Guess, Calculation of perforated plate liner parameters from specified acoustic resistance and reactance, J. Sound Vib. 40(1) (1975) 119-137.

[3] M.L. Munjal, Acoustics of ducts and mufflers with application to exhaust and ventilation system design, John Wiley \& Sons, (1987).

[4] M.G. Jones, M.B. Tracy, W.R. Watson, and T.L. Parrot, Effect of liner geometry on acoustic impedance, 8th AIAA/CEAS Aeroacoustics Conference $\mathcal{E}$ Exhibit, 17-19 June 2002, Breckenridge, CO, USA, AIAA Paper 2002-2446.

[5] L. Cremer, Theorie des Luftschall-Dämpfung im Rechteckkanal mit schluckender Wand und das sich dabei ergebende höchste Dämpfungsmaß [Theory regarding the attenuation of sound transmitted by air in a rectangular duct with an absorbing wall, and the maximum attenuation constant produced during this process], Acustica 3 (1953) 249-263.

[6] B.J. Tester, The optimization of modal sound attenuation in ducts, in the absence of mean flow, J. Sound Vib. 27(4) (1973) 477-513.

[7] B.J. Tester, The propagation and attenuation of sound in lined ducts containing uniform or "plug" flow, J. Sound Vib. $28(2)$ (1973) 151-203.

[8] W.R. Watson, M.G. Jones, S.E. Tanner, and T.L. Parrot, Validation of a numerical method for extracting liner impedance, AIAA J. 34(3) (1996) 548-554.

[9] R.Troian, D. Dragna, C. Bailly, and M.A. Galland, Broadband liner impedance eduction for multimodal acoustic propagation in the presence of a mean flow, J. Sound Vib. 392 (2017) 200-216.

[10] H.F. Olson, E.G. May, Electronic Sound Absorber, J. Acous. Soc. Am. 25 (1953) 1130-1136.

[11] D. Guicking and E. Lorentz, An active sound absorber with porous plate, J. Vib. Acoust. Stress Reliability Des. 106 (1984) $389-392$.

[12] M. Furstoss, D. Thenail, and M.A. Galland, Surface impedance control for sound absorption: direct and hybrid passive/active strategies, J. Sound Vib. 203(2) (1997) 219-236.

[13] M.A. Galland, N. Sellen, and M. Cuesta, Hybrid passive/active absorbers for flow ducts, Appl. Acoust. 66 (2005) $691-708$.

[14] N. Sellen, M. Cuesta, and M.A. Galland, Noise reduction in a flow duct: implementation of a hybrid passive/active solution, J. Sound Vib. 297 (2006) 492-511.

[15] B. Betgen, M.A. Galland, E. Piot, and F. Simon, Implementation and non-intrusive characterization of a hybrid active-passive liner with grazing flow, Appl. Acoust. 73 (2012) 624-638.

[16] R.E. Kraft and K.B. Kontos, Active acoustic liner, US Patent, US 5,498,127 A (1996)

[17] H. Zhao and X. Sun, Active control of wall acoustic impedance, AIAA J. 37(7) (1999) 825-831.

[18] S. Horowitz, T. Nishida, L.N. Cattafesta, and M. Sheplak, Characterization of a compliant-backplate Helmholtz resonator for an electromechanical acoustic liner, International J. Aeroacoustics 1 (2) (2002) 283-205.

[19] A.S. Hersh and J. Tso, Extended frequency range Helmholtz resonators, US Patent, US 5,119,427 (1992).

[20] C.A. Parente, N. Arcas, B.E. Walker, A.S. Hersh, and E.J. Rice, Hybrid active/passive jet engine noise suppression system, NASA/CR 1999-208875 (1999).

[21] M. Collet, P. David, and M. Berthillier, Active acoustical impedance using distributed electrodynamical transducers, J. Acoust. Soc. Am. 125(2) (2009) 882-894.

[22] H. Lissek, Shunt loudspeaker technique for use as acoustic liner, 38th International Congress and Exposition on Noise Control Engineering, INTER-NOISE 2009, 23-26 August, Ottawa, Canada.

[23] R. Boulandet and H. Lissek, Optimization of electroacoustic absorbers by means of designed experiments, Appl. Acoust. 71 (2010) 830-842.

[24] H. Lissek, R. Boulandet, and R. Fleury, Electroacoustic absorbers: bridging the gap between shunt loudspeakers and active sound absorption, J. Acoust. Soc. Am. 129(5) (2009) 2968-2978.

[25] R. Boulandet and H. Lissek, Toward broadband electroacoustic resonators through optimized feedback control strategies, J. Sound Vib. 333 (2014) 4810-4825.

[26] R. Boulandet, E. Rivet, and H. Lissek, Sensorless electroacoustic absorber through synthesized electrical impedance, Acta Acust. united Acust. 102 (2016) 696-704

[27] E. Rivet, S. Karkar, and H. Lissek, Broadband low-frequency electroacoustic absorbers through hybrid sensor-/shunt-based impedance control, IEEE Transactions on Control Systems Technology 25 (1) (2017) 63-72. 
[28] S.H. Ko, Sound attenuation in lined rectangular ducts with flow and its application to the reduction of aircraft engine noise, J. Acoust. Soc. Am. 50(6) (1971) 1418-1432.

[29] R.E. Motsinger and R.E. Kraft, Design and performance of duct acoustic treatment, Aeroacoustics of flight vehicles: Theory and Practice, H.H. Hubbard (Ed.), NASA RP-1258, 2 (1991) 165-206.

[30] C.K.W. Tam and L. Auriault, Time domain impedance boundary for computational aeroacoustics, AIAA J. 34(5) (1996) 917-923.

[31] S.W. Rienstra, Impedance models in time domain including the extended Helmholtz resonator model, 12th AIAA/CEAS Aeroacoustics Conference, 8-10 May 2006, Cambridge, USA, AIAA Paper 2006-2686.

[32] C. Richter, F.H. Thiele, X. Li, and M. Zhuang, Comparison of time-domain impedance boundary conditions for lined duct flows, AIAA J. 45(6) (2007) 1333-1345.

[33] W.K. Chen, The Circuits and Filters Handbook, CRC Press, Boca Raton, (1995).

[34] D.L. Lansing and W.E. Zorumski, Effect of wall admittance changes on duct transmission and radiation of sound, J. Sound Vib. 27(1) (2016) $85-100$.

[35] K.U. Ingard, Influence of fluid motion past a plane boundary on reflection, absorption, and transmission, J. Acoust. Soc. Am. 31(7) (1959) 1035-1036.

[36] M.K. Myers, On the acoustic boundary condition in the presence of flow, J. Sound Vib. 71(8) (1980) $429-434$.

[37] D. Givoli, B. Neta, High-order non-reflecting boundary scheme for time-dependent waves, J. Comp. Phys. 186 (2003) 24-46.

[38] V. Pagneux, N. Amir, and J. Kergomard, A study of wave propagation in varying cross-section waveguides by modal decomposition. Part I. Theory and validation, J. Acoust. Soc. Am. 100(4) (196) 2034-2048.

[39] P.E. Doak, Excitation, transmission and radiation of sound from source distributions in hard-walled ducts of finite length (I): the effect of duct cross-section geometry and source distribution space-time pattern, J. Sound Vib. 31(1) (1980) 1-72.

[40] C.L. Morfey, Sound transmission and generation in ducts with flow, J. Sound Vib. 14 (1) (1971) 37-55

[41] P. Joseph, C.L. Morfey, C.R. Lowis, Multi-mode sound transmission in duct with flow, J. Sound Vib. 264 (2003) 523-544.

[42] W. Neise, W. Frommhold, F.P. Mechel, and F. Holste, Sound power determination in rectangular flow ducts, J. Sound Vib. 174(2) (1994) 201-237.

[43] ASTM E477-13e1 Standard test method for laboratory measurements of acoustical and airflow performance of duct liner materials and prefabricated silencers, ASTM International, West Conshohocken, PA (2013). 